\title{
Replication Variance of African and Asian Lineage Zika Virus Strains in Different Cell Lines, Mosquitoes and Mice
}

\author{
Tey Putita Ou ${ }^{1,+}+$, Heidi Auerswald ${ }^{1,+}\left(\mathbb{D}\right.$, Saraden In ${ }^{1}$, Borin Peng ${ }^{1}$, Senglong Pang ${ }^{1}$, Sébastien Boyer ${ }^{2}$,

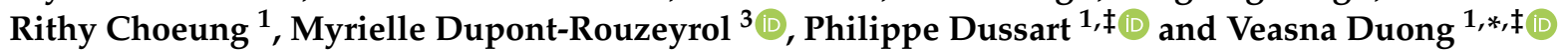 \\ 1 Virology Unit, Institut Pasteur du Cambodge, Institut Pasteur International Network, \\ Phnom Penh 12156, Cambodia; oteyputita@pasteur-kh.org (T.P.O.); hauerswald@pasteur-kh.org (H.A.); \\ insaraden@pasteur-kh.org (S.I.); borinpeng@gmail.com (B.P.); psenglong@pasteur-kh.org (S.P.); \\ rithychoeung88@yahoo.com (R.C.); pdussart@pasteur.mg (P.D.) \\ 2 Medical Entomology Unit, Institut Pasteur du Cambodge, Institut Pasteur International \\ Network, Phnom Penh 12156, Cambodia; sboyer@pasteur-kh.org \\ 3 URE Dengue and Arboviruses, Institut Pasteur in New Caledonia, Institut Pasteur International Network, \\ Nouméa 98800, New Caledonia; mdupont@pasteur.nc \\ * Correspondence: dveasna@pasteur-kh.org \\ + These authors contributed equally to this work. \\ $\ddagger$ Joint Senior Authors—-these authors contributed equally to this work.
}

Citation: Ou, T.P.; Auerswald, H.; In S.; Peng, B.; Pang, S.; Boyer, S.; Choeung, R.; Dupont-Rouzeyrol, M.; Dussart, P.; Duong, V. Replication Variance of African and Asian Lineage Zika Virus Strains in Different Cell Lines, Mosquitoes and Mice. Microorganisms 2021, 9, 1250. https://doi.org/10.3390/ microorganisms 9061250

Academic Editors: Dániel Cadar and Miguel A. Martín-Acebes

Received: 18 April 2021

Accepted: 25 May 2021

Published: 9 June 2021

Publisher's Note: MDPI stays neutral with regard to jurisdictional claims in published maps and institutional affiliations.

Copyright: (c) 2021 by the authors. Licensee MDPI, Basel, Switzerland. This article is an open access article distributed under the terms and conditions of the Creative Commons Attribution (CC BY) license (https:// creativecommons.org/licenses/by/ $4.0 /)$.

\begin{abstract}
Since the epidemic in 2007, studies on vector competence for Zika virus (ZIKV) have intensified, showing that the transmission efficiency varies depending on the vector population, ZIKV strain, and dose of the infectious blood meal. In this study, we aimed to investigate the replication of African and Asian ZIKV strains in vitro and in vivo in order to reveal their phenotypic differences. In addition, we investigated the vector competence of Cambodian Aedes aegypti (Ae. aegypti) mosquitoes (urban and rural) for these ZIKV strains. We observed a significantly higher pathogenicity of the African ZIKV strain in vitro (in mosquito and mammalian cells), and in vivo in both Ae. aegypti and mice. Both mosquito populations were competent to transmit ZIKV as early as 7 days p.i., depending on the population and the ZIKV strain. Ae. aegypti from rural habitats showed significant higher transmission and survival rates than those from urban. We observed the highest transmission efficiency for the African ZIKV isolate (93.3\% 14 days p.i.) and for the Cambodian ZIKV isolate ( $80 \% 14$ days p.i.). Overall, our results highlight the phenotypic differences of the ZIKV lineages and the potential risk of ZIKV transmission by Ae. aegypti mosquitoes. Further investigations of Cambodian mosquito species and ZIKV specific surveillance in humans is necessary in order to improve the local risk assessment.
\end{abstract}

Keywords: Zika virus; African lineage; Asian lineage; vector competence; neonatal mouse infection

\section{Introduction}

Zika virus (ZIKV) is a flavivirus mainly transmitted by Aedes mosquito species, representing an example of the emergence of a new arboviral disease. It was first isolated in Uganda in 1947 [1], and for 60 years, infections with this virus were rarely reported. In Asia, ZIKV was first isolated in 1966 in Malaysia from Aedes (Ae.) aegypti mosquitoes [2], and the first human infections were reported in 1977 in Central Java, Indonesia [3]. Serological evidence for ZIKV transmission in Southeast Asia was found in surveys in the early 1950s in the Philippines, Malaysia, Thailand, and Vietnam [4]. The first major outbreak was documented in 2007 on Yap island (Micronesia), where over 70\% of the population was infected [5]. In 2010, ZIKV was isolated in Cambodia from a single sample through a fever surveillance [6], but no further infections were reported at that time. In 2013-2014, a large ZIKV outbreak occurred in French Polynesia [5]. The virus spread further over the Pacific islands and in 2015, a massive epidemic of ZIKV hit the Americas and became 
an international public health emergency. For the first time, this outbreak included reports of increased numbers of congenital microcephaly associated with maternal ZIKV infections [7].

Epidemiological and phylogenetic studies discovered that ZIKV strains were grouped by geographic origin, namely, the initial African lineage and the diverged Asian lineage that spread in the 1950s through Southeast Asia [8]. Within this Asian lineage, isolates from the recent ZIKV outbreaks in the Americas clustered together in an American subgroup, whereas the older Asian strains formed a separate subgroup (Supplementary Figure S1) [9].

Because of the significant public health risk, studies on vector competence for ZIKV intensified globally over the last years [10]. The determined transmission efficiency varies depending on the vector population, the ZIKV strain, and the viral titer of the infectious blood meal. Therefore, these data are difficult to extrapolate, and investigations with local vector populations are necessary for more targeted risk assessment and modelling [11-13]. Besides the in vivo experiments in mosquitoes, mammals have been utilized to further understand the biology and pathogenicity of ZIKV [14]. Murine models are valuable tools, and have been utilized to demonstrate the neurotropism and pathogenicity of ZIKV [15].

In Cambodia, the detection of ZIKV relies on incidental findings, because a ZIKV surveillance program for human or mosquito infections is missing. A retrospective prevalence analysis in samples collected from DENV suspected cases from the years 2007-2016 were tested for the presence of ZIKV by RT-PCR [16]. This revealed a low prevalence of $0.2 \%(n=5)$ among the 2400 samples tested. The main ZIKV vector, Ae. aegypti, is widely distributed in Cambodia [16]. Moreover, Cambodia also has a long history of simultaneous circulation of all four DENV serotypes, with outbreaks occurring frequently every 3-4 years [17]. However, the Dengue National Surveillance System is not suitable to monitor ZIKV infections, as the majority of these infections are asymptomatic and the DENV surveillance focusses on clinical febrile illness in hospitalized children. Thus, the evaluation of the vector competence of Cambodian mosquitoes for ZIKV is important for the risk assessment of the re-emergence of ZIKV in Cambodia.

The aim of our study was the investigation of three ZIKV strains from the two lineages - one belonging to the African lineage and two to the Asian lineage-for their replication in different in vitro and in vivo models. We analyzed their growth in different mosquito cells and in a mammalian cell line. In vivo, we determined the vector competence of urban and rural Cambodian field-collected Ae. aegypti populations for the different ZIKV strains and compared the virulence and pathogenesis of the three ZIKV strains in a murine model.

\section{Materials and Methods}

\subsection{Cells}

The simian Vero cell line (ATCC CCL-81) was maintained in Dulbecco's modified Eagle medium (DMEM; Sigma-Aldrich, St. Louis, MO, USA) supplemented with 10\% fetal bovine serum (FBS; Gibco, Waltham MA, USA) and antibiotics (100 units/mL of penicillin and $100 \mu \mathrm{g} / \mathrm{mL}$ of streptomycin; Gibco) at $37^{\circ} \mathrm{C}$ under a $5 \% \mathrm{CO}_{2}$ humidified atmosphere. The three mosquito cell lines used, Aag2 (Ae. Aegypti, provided by Julien Pompon, Duke-NUS, Singapore), AP-61 (Ae. Pseudoscutellaris, Centre National de Référence des arbovirus, Institut Pasteur, Paris, France), and C6/36 (Ae. Albopictus, ATCC CRL-166), were maintained in Leibovitz's L-15 medium (Sigma-Aldrich) supplemented with 10\% FBS, antibiotics, $2 \mathrm{mM}$ L-glutamine (Gibco), and $10 \%$ tryptose phosphate (Gibco) at $28{ }^{\circ} \mathrm{C}$ under a humidified atmosphere.

\subsection{Viruses}

The African lineage ZIKV strain HD78788 (GenBank: KF383039), originally isolated from a patient in Senegal in 1991 [18], was produced in AP-61 cells. This virus strain is highly adapted to cultivation in various cell lines and the passage history is unknown. The Asian lineage ZIKV strains NC-2014-5132 (GenBank: SRR5309452) and FSS13025 (GenBank: 
KU955593) were grown in C6/36 cells. Strain NC-2014-5132 was isolated from a patient in April 2014 in New Caledonia, and passaged five times in Vero cells and eight times in C6/36 cells before its use in this study. Strain FSS13025 was isolated on Vero cells from a febrile patient in Cambodia in 2010 [6] and passaged six times in C6/36 cells before use in this study. Virus culture supernatants were stored at $-80{ }^{\circ} \mathrm{C}$ before titration via FFA and their further use in infection experiments of cells, mosquitoes, and mice.

\subsection{Mosquito Infection}

Mosquito populations from urban Phnom Penh (urban-PP) and from rural Kampong Cham (rural-KC) were collected at the end of rainy season (December 2017) as larvae or pupae, using black plastic cups filled with water and ovitraps (Green Ornament, Osaka, Japan). Field-collected eggs and larvae were reared and fed with half a teaspoon of grounded fish food daily until the emergence of adults (F0 generation). Adult mosquitoes were kept in cages at a temperature of $28 \pm 1{ }^{\circ} \mathrm{C}$ with $12 \mathrm{~h}: 12 \mathrm{~h}$ light:dark cycle, $75 \pm 2.5 \%$ relative humidity, and with a $10 \%$ sucrose solution. Additionally, the females were provided with lab-reared mice for blood meal for $1 \mathrm{~h}$ once a week. Adult mosquitoes of the F0 generation were identified morphologically according to the identification key for mosquitoes in Southeast Asia countries [19-21]. Only clearly identified Ae. aegypti were used for rearing of the F1 generation. Mosquito eggs were collected and hatched in dechlorinated tap water. Emerged larvae were divided into groups of 200 larvae per $1 \mathrm{~L}$ water, and were fed daily with 1-2 pellets of rabbit food (Kaytee, Chilton, WI, USA). Adult female mosquitoes of the F1 generation were maintained solely on a $10 \%$ sucrose solution before infectious blood meal feeding.

All F1 females aged between 3-5 days were starved by removing the sucrose $24 \mathrm{~h}$ before artificial blood meal. For the infection, mosquitoes were sorted into cups containing 30 mosquitoes per cup, and were transferred into the BSL3 laboratory. Artificial blood meals contained $1.4 \mathrm{~mL}$ of washed human erythrocytes, viral suspension (final concentration: $\sim 10^{6} \mathrm{FFU} / \mathrm{mL}$ ), and $5 \mathrm{mM}$ adenosine triphosphate (ATP, Sigma-Aldrich). The ZIKV concentration of the blood-meal was determined by titration on the same day via FFA. For each mosquito population, 300-360 female mosquitos were used for each ZIKV strain. After $30 \mathrm{~min}$ of feeding on a pig intestinal membrane attached to feeders of a Hemotek blood-feeding system (Hemotek, UK), the blood-fed mosquitoes were separated, transferred into new cups, and were maintained at $28 \pm 1^{\circ} \mathrm{C}$, with a $12 \mathrm{~h}: 12 \mathrm{~h}$ light:dark cycle and $80 \%$ humidity, and were fed daily with $10 \%$ sucrose.

On days 7, 10, 14, and 21 after infection, 30 mosquitoes per virus strain and mosquito population were cold-anesthetized. Salivation was forced by inserting the proboscis for 30 min into a $20 \mu \mathrm{L}$ tip containing $5 \mu \mathrm{L}$ of FBS. Afterwards, the saliva-containing FBS was mixed with $45 \mu \mathrm{L}$ DMEM supplemented with antibiotics and $2.5 \mu \mathrm{g} / \mathrm{mL}$ Amphotericin $\mathrm{B}$ (Gibco). The head, legs, and wings of each individual mosquito were stored together in $400 \mu \mathrm{L}$ phosphate buffered saline (PBS) supplemented with 10\% FBS, antibiotics, and Amphotericin B and ceramic beads until homogenization. The remaining body (abdomen and thorax) of each female was processed in the same way. The homogenization was performed using a MagNA Lyser (Roche, Basel, Switzerland) at $6500 \mathrm{rpm}$ for $50 \mathrm{~s}$.

\subsection{Mice}

As a result of availability, the Swiss mice (male and female) used in our study were maintained under pathogen-free and hygiene conditions in the Institute Pasteur in Cambodia in BSL2 facility. One day-old Swiss mice $(n=18-20$ per group) were infected intracerebrally with ZIKV in order to compare the pathogenicity of different ZIKV strains on mice. The inoculum contained $50 \mu \mathrm{L}$ of ZIKV suspension $\left(10^{5} \mathrm{FFU} / \mathrm{mL}\right)$ or PBS as the control. The weight and clinical symptoms were monitored daily over 21 days. The level of clinical symptoms was scored as follows: (0) healthy, (1) fever, (2) weaker and more emaciated, (3) limb weakness and back bending, (4) hind-limb or fore-limb paralysis and tremors, and (5) death. Mice infected with NC-2014-5132 and FSS13025 were euthanized at 
day 7, between day 8 and 10, and at day 21 p.i. with the cervical dislocation method [22], in accordance to the date of mortality observed in mice infected by HD78788 virus. After death or euthanasia, the blood samples and organs tissues were collected. Blood was obtained via cotton swabs and was stored in $400 \mu \mathrm{L}$ PBS. The organs (brain, heart, intestines, liver, kidney, lung, pancreas, thorax, and primary sex organs) were stored individually in $400 \mu \mathrm{L}$ PBS, and homogenized with MagNA Lyser at $6500 \mathrm{rpm}$ for $50 \mathrm{~s}$ before use.

\subsection{Real-Time RT-qPCR}

The amount of ZIKV titer in the mosquito and mouse organ homogenates was determined by real-time RT-qPCR [23] with RNA extracted using the QIAamp Viral RNA Mini kit (Qiagen, Hilden, Germany).

\subsection{Focus Forming Assay}

The titer of infectious virus was determined by focus forming assay (FFA) and was expressed as focus forming units per milliliter (FFU/mL), as described previously [23]. Titrations were always performed in duplicate. Infected cells visible as foci were automatically counted using an AID ELISpot Reader (Autoimmun Diagnostika GmbH, Strassberg, Germany).

\subsection{Cell Infection}

For the ZIKV growth kinetics, the respective cells (Aag2, C6/36, and Vero) were seeded in 48-well plates with a density of $5 \times 10^{4}$ cells/well. The following day, the ZIKV infection was done with different multiplicities of infection (MOI: 0.01, 0.1, and 1) for $1 \mathrm{~h}$ at the growth conditions of the respective cell lines. ZIKV stocks used for inoculation were back-titrated by FFA on the same days to ensure correct MOIs. Afterwards, the virus inoculum was replaced by supplemented medium of the respective cell line, as described above with a decreased FBS content (5\%). Every $24 \mathrm{~h}$, samples were taken in duplicate and were freshly titrated by FFA. CPE was observed using brightfield microscopy, but was not further quantified.

\subsection{Ethics Statement}

During this study, we followed the World Animal Health Organization (OIE) guiding principles on animal welfare included in the OIE Terrestrial Animal Health Code [24]. Mouse experiment were approved by the National Animal Health and Production Research Institute (NAHPRI) from General Directorate for Animal Health and Production (GDAHP), Ministry of Agriculture, Forestry, and Fisheries. Written consent was obtained from the blood donor volunteers before sampling.

\subsection{Statistical Analysis}

All of the statistical analyses were performed using GraphPad Prism for Windows, version 7.0.1 (GraphPad Software, Inc., La Jolla, CA, USA), and a level for significance of $\alpha<0.05$. The statistical test used for each experiment is indicated in the respective results section or figure descriptions.

\section{Results}

\subsection{ZIKV Growth Kinetic in Mammalian and Mosquito Cell Lines}

We analyzed the replication of the three ZIKV strains in the Vero cell line (Figure 1A-C) and the mosquito cell lines C6/36 (Ae. albopictus; Figure 1D-F) and Aag2 (Ae. aegypti; Figure 1G-I). The mosquito cell lines that were used were Ae. aegypti and Ae. Albopictus, which are both relevant vectors in Cambodia. The Ae. pseudoscutellaris cell line AP-61 was not used for the ZIKV growth curves, as this mosquito species is spread over the South Pacific islands [25] and is therefore not a relevant vector for the Greater Mekong region. The simian cell line was used for comparison, as flavivirus replication has been well studied in these cells $[26,27]$. The two Asian lineage viruses replicated similarly in all cell lines at all 
different MOIs. The African strain HD78788 exhibited a cytopathic effect (CPE) from day 2 p.i. in the mosquito cells (red squares in Figure 1D-F for C6/36, and Figure 1G-H for Aag2) and from day 5 p.i. in the Vero cells. Overall, the three virus strains grew similarly in the simian Vero cells. HD78788 grew to the highest titer in Vero cells inoculated with MOI 0.01 (Figure $1 \mathrm{~A}$; peak titer $3.38 \times 10^{6} \mathrm{ffu} / \mathrm{mL}$ on day 4 p.i.) compared with NC-2014-5132 (6.67 $\times 10^{5} \mathrm{ffu} / \mathrm{mL}, p=0.018$; two-way ANOVA analysis with Tukey's correction) and FSS13025 $\left(3.93 \times 10^{5} \mathrm{ffu} / \mathrm{mL} p=0.0097\right)$. Despite the CPE only seen for the cells infected with HD787888, we observed differences in the max one log in the viral titers of the different ZIKV strains grown in the Ae. albopictus cell line C6/36. Compared with strain FSS13025, we observed significantly lower viral titers of HD78788 at MOI 0.01 on day 7 p.i. $(p=0.0072$; Figure 1D) and at MOI 0.1 on day 4 p.i. $(p=0.0357$; Figure 1E), day 6 p.i. $(p=0.0219)$, and day 7 p.i. $(p=0.0183)$. However, HD78788 grew to the higher titer in C6/36 cells inoculated with a high virus load of MOI 1 (Figure $1 \mathrm{~F}$; peak titer $1.92 \times 10^{7} \mathrm{ffu} / \mathrm{mL}$ on day 4 p.i.) compared with NC-2014-5132 (4.18 $\left.\times 10^{6} \mathrm{ffu} / \mathrm{mL}, p=0.0063\right)$ and FSS13025 $\left(1.00 \times 10^{7} \mathrm{ffu} / \mathrm{mL} p=0.0938\right)$. In contrast, for the growth differences in the Ae. aegypti cells (Aag2) infected with HD78788, the CPE was so strong that the virus replication was strongly inhibited and was therefore significant lower at several time points under different MOIs. The African strain HD78788 grew significantly slower, e.g., demonstrated at MOI 1 4 days p.i. (titer $8.13 \times 10^{5} \mathrm{ffu} / \mathrm{mL}$; compared with NC-2014-5132 titer $9.80 \times 10^{6} \mathrm{ffu} / \mathrm{mL}$, $p=0.0251$; compared with FSS13025 titer $\left.1.15 \times 10^{7} \mathrm{ffu} / \mathrm{mL}, p=0.0085\right)$. Overall, all three ZIKV strains were replicated similarly in simian cells. We observed CPE for only the African ZIKV strain, and the differences in growth were especially prominent in the Ae. aegypti cells.

\subsection{Vector Competence of Cambodian Ae. aegypti Mosquitoes}

As we observed significantly higher growth rates for the Asian lineage ZIKV strains in $A$ e. aegypti cells when we compared the vector competence of Cambodian Ae. aegypti mosquitoes to these ZIKV strains. We conducted the investigation on two different mosquito populations, one from urban Phnom Penh (PP) and one from rural Kampong Cham (KC). For all of these experiments, ZIKV was detected and quantified using real-time RT-qPCR, and the presence of live ZIKV in saliva was confirmed by FFA.

\subsubsection{ZIKV Infection Rate}

The infection rates, defined as ZIKV-positive bodies among all blood-fed mosquitoes, were similar (average $\geq 93 \%$ ) at all-time points (day 7 p.i., 10 p.i., 14 p.i., and 21 p.i.) for all ZIKV strains (Figure 2A,B) for both urban-PP and rural-KC mosquito populations (Supplementary Tables S1 and S2). 

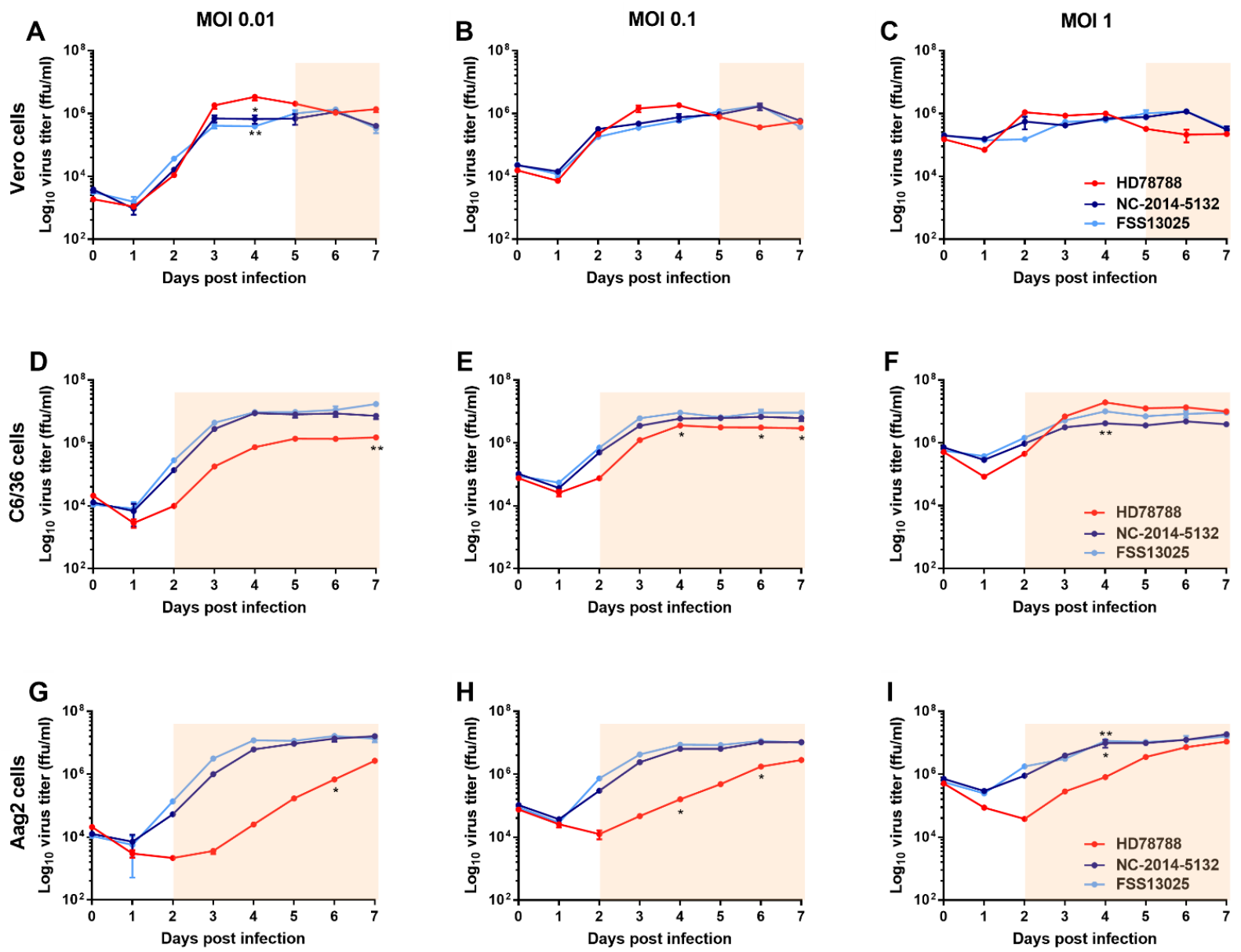

Figure 1. Zika virus (ZIKV) growth kinetic in different cell lines. ZIKV HD78788 (red), NC-2014-5132 (dark blue), and FSS13025 (light blue) were analyzed for their growth in vero (A-C) and mosquito cells (C6/36: (D-F); Aag2: (G-I)). Cells were infected with different multiplicity of infection (MOI): 0.01 (A,D,G), 0.1 (B,E,H), and 1 (C,F,I). Values represent means \pm standard deviation of duplicate experiments. Cytopathic effect was observed in cells infected with HD787888 (light red background squares). Statistical differences determined with two-way ANOVA analysis (Tukey's multiple comparison correction) are marked by asterisks: ${ }^{*} p<0.05$ and ${ }^{* *} p<0.01$.

\subsubsection{ZIKV Dissemination Rate}

The dissemination rate of ZIKV HD78788 in urban-PP mosquitoes was significantly higher 7 and 10 days p.i. (100\%, Figure 2C) than that of NC-2014-5132 (day 7 p.i.: $80.0 \%$, $p=0.0237$; day 10 p.i.: $82.8 \%, p=0.0237$ ) and FSS13025 (day 7 p.i.: $26.7 \%, p<0.0001$; day 10 p.i.: $55.2 \%, p<0.0001)$. The dissemination rates were also different between the Asian ZIKVs. Urban-PP mosquitoes infected with FSS13025 had significantly lower dissemination rates than mosquitoes infected with NC-2014-5132 at day 7 p.i. $(p<0.0001)$ and at day 10 p.i. $(p=0.0454)$. At days 14 and 21 p.i., the dissemination rates were $\geq 97 \%$ for all ZIKVs in urban-PP mosquitoes, with no significant differences (Supplementary Table S1). The dissemination rates in rural-KC mosquitoes were homogenously high at all-time points and for all ZIKVs ( $\geq 93 \%$; Figure 2D; Supplementary Table S2). 


\section{Phnom Penh}

A
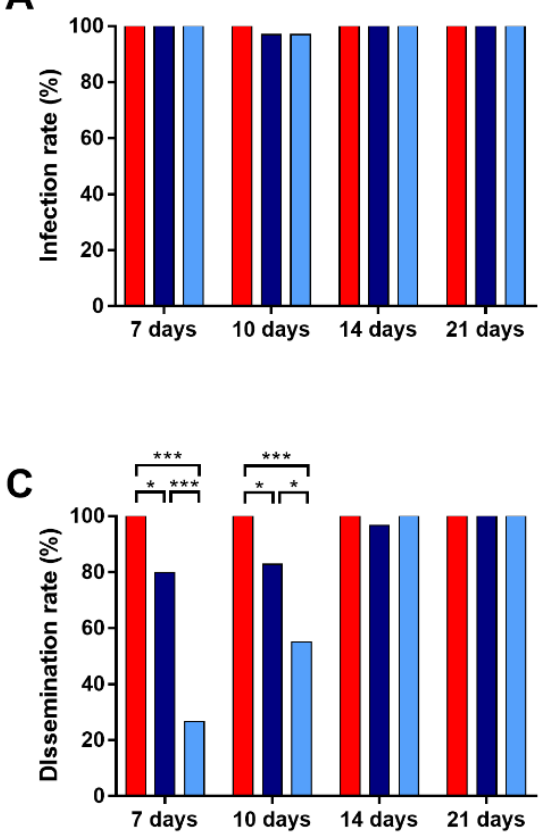

E

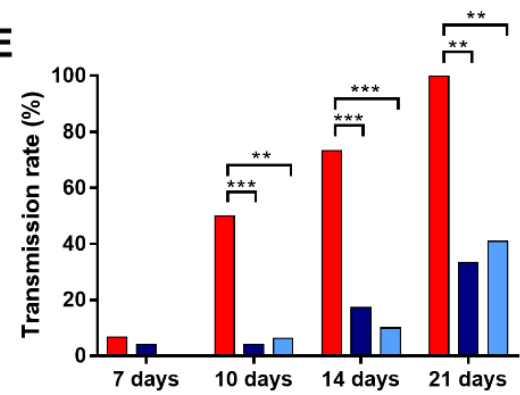

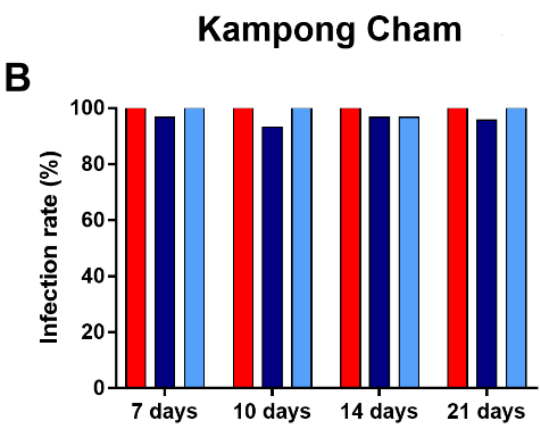

D
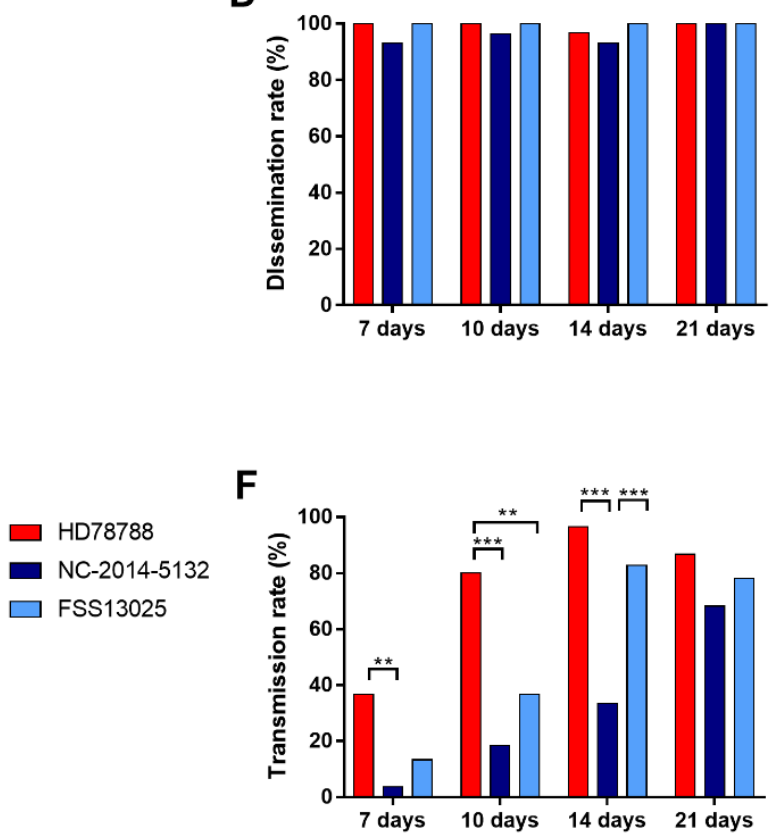

$\mathbf{F}$

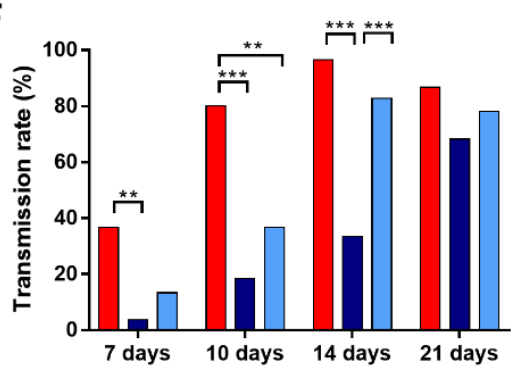

Figure 2. Infection, dissemination, and transmission rates of Cambodian Ae. aegypti mosquitoes. Ae. aegypti populations from Phnom Penh (A,C,E) and Kampong Cham (B,D,F) were infected with ZIKV HD78788 (red), NC-2014-5132 (dark blue), or FSS13025 (light blue). Infection rates (A,B) are expressed as the percentage of mosquitoes with ZIKV-positive bodies among the total number of blood-fed mosquitoes. Dissemination rates $(\mathbf{C}, \mathbf{D})$ are expressed as the percentage of mosquitoes with ZIKV-positive head, legs, and wings among the mosquitoes with ZIKV-positive bodies. Transmission rates (E,F) are expressed as percentage of mosquitoes with ZIKV-positive saliva among mosquitoes with ZIKV-positive bodies. Statistical differences determined with Fisher's exact test are marked by asterisks: ${ }^{*} p<0.05,{ }^{* *} p<0.01$, and ${ }^{* *} p<0.001$.

\subsubsection{ZIKV Transmission Rate}

The transmission rates, determined as mosquitoes with ZIKV-positive saliva among mosquitoes with disseminated infection, were higher for the African ZIKV HD78788 compared with the two Asian isolates at all-time points (urban-PP: Figure 2E, Supplementary Table S1; rural-KC: Figure 2F, Supplementary Table S2). ZIKV was detected in the saliva of urban-PP mosquitoes as early as day 7 p.i. for HD78788 (6.7\%) and NC-2014-5132 (4.2\%) strains, and from day 10 p.i. for FSS13025 (6.3\%). The transmission rate for HD78788 increased over the following time points (day 10 p.i.: $50.0 \%$; day 14 p.i.: $73.3 \%$ ) to $100 \%$ at day 21 p.i., and was significantly higher than NC-2014-5132 (day 10 p.i.: $4.2 \%, p=0.0002$; day 14 p.i.: $17.2 \%, p<0.0001$; day 21 p.i.: 33.3\%, $p=0.0019$ ) and FSS13025 (day 10 p.i.: $6.3 \%, p=0.0033$; day 14 p.i.: 10.0\%, $p<0.0001$; day 21 p.i.: $40.9 \%, p=0.0084)$. For rural-KC mosquitoes, ZIKV was detected at higher rates, with transmission rates significantly higher for HD78788 on day 7 p.i. (36.7\%), day 10 p.i. (80.0\%), and day 14 p.i. (96.6\%) than for 
NC-2014-5132 (day 7 p.i.:3.7\%, $p=0.0028$; day 10 p.i.: $18.5 \%, p<0.0001$; day 14 p.i.: $33.3 \%$, $p<0.0001$ ). The transmission rate of HD78788 was also significantly higher when compared with FSS13025 on day 10 p.i. $(36.7 \%, p=0.0014)$. The transmission rate of FSS13025 increased notably over different time points and was significantly higher than the rate for NC-2014-5132 at day 14 p.i. $(p=0.0003)$.

\subsubsection{ZIKV Transmission Efficiency}

The ZIKV transmission efficiency represents the number of mosquitoes with ZIKVpositive saliva among the total number of tested mosquitoes. The transmission efficiency rates were similar to the transmission rates. However, a comparison between the two mosquito populations revealed significantly higher transmission efficiencies for the ruralKC mosquitoes (Table 1).

Table 1. ZIKV transmission efficiency of two Cambodian Ae. aegypti populations.

\begin{tabular}{|c|c|c|c|c|c|c|c|}
\hline \multirow{2}{*}{$\begin{array}{l}\text { Days after } \\
\text { Infection }\end{array}$} & \multirow{2}{*}{$\begin{array}{c}\text { Mosquito } \\
\text { Population (Origin) }\end{array}$} & \multicolumn{2}{|c|}{ HD78788 } & \multicolumn{2}{|c|}{ NC-2014-5132 } & \multicolumn{2}{|c|}{ FSS13025 } \\
\hline & & $\begin{array}{c}\text { Transmission } \\
\text { Efficiency * }\end{array}$ & $p$ Value ${ }^{\#}$ & $\begin{array}{c}\text { Transmission } \\
\text { Efficiency * }\end{array}$ & $p$ Value $\#$ & $\begin{array}{c}\text { Transmission } \\
\text { Efficiency }\end{array}$ & $p$ Value $\#$ \\
\hline \multirow{2}{*}{7} & urban-PP & $6.7 \% \quad(2 / 30)$ & \multirow{2}{*}{0.0102} & $3.3 \% \quad(1 / 30)$ & \multirow{2}{*}{1.000} & $0 \% \quad(0 / 30)$ & \multirow{2}{*}{0.1124} \\
\hline & rural-KC & $36.7 \% \quad(11 / 30)$ & & $3.3 \% \quad(1 / 30)$ & & $13.3 \% \quad(4 / 30)$ & \\
\hline \multirow{2}{*}{10} & urban-PP & $50.0 \% \quad(15 / 30)$ & \multirow{2}{*}{0.0292} & $3.3 \% \quad(1 / 30)$ & \multirow{2}{*}{0.1945} & $3.3 \% \quad(1 / 30)$ & \multirow{2}{*}{0.0025} \\
\hline & rural-KC & $80.0 \% \quad(24 / 30)$ & & $16.7 \% \quad(5 / 30)$ & & $36.7 \% \quad(11 / 30)$ & \\
\hline \multirow{2}{*}{14} & urban-PP & $73.3 \% \quad(22 / 30)$ & \multirow{2}{*}{0.0797} & $16.7 \% \quad(5 / 30)$ & \multirow{2}{*}{0.3604} & $10.0 \% \quad(3 / 30)$ & \multirow{2}{*}{$<0.0001$} \\
\hline & rural-KC & $93.3 \% \quad(28 / 30)$ & & $30.0 \% \quad(9 / 30)$ & & $80.0 \% \quad(24 / 30)$ & \\
\hline \multirow{2}{*}{21} & urban-PP & $100 \% \quad(7 / 7)$ & \multirow{2}{*}{1.000} & $33.3 \% \quad(10 / 30)$ & \multirow{2}{*}{0.0283} & $40.9 \% \quad(9 / 22)$ & \multirow{2}{*}{0.0031} \\
\hline & rural-KC & $86.7 \% \quad(13 / 15)$ & & $68.2 \% \quad(15 / 23)$ & & $78.0 \% \quad(39 / 50)$ & \\
\hline
\end{tabular}

* Number of mosquitoes with ZIKV positive saliva/total number of blood-fed mosquitoes. ${ }^{\#}$ Two-tailed Fisher's exact test; urban-PP—urban Phnom Penh; rural-KC—rural Kampong. Cham.

\subsection{ZIKV Viral Load in Infected Mosquitoes}

We also investigated the viral load in the artificially infected mosquitoes. We observed an increase in the viral load in the saliva over time in both populations (urban-PP: Figure 3A; rural-KC: Figure 3B). We observed the same pattern for the dissemination into the legs, wings, and head (Supplementary Figure S2A,B), whereas the viral load in the bodies of the mosquitoes stayed constant (Supplementary Figure S2C,D). However, the viral load in the saliva, as well as the legs and wings, was higher for the rural-KC mosquitoes. As seen for the transmission rates and the transmission efficiency, the viral load in the saliva was significantly higher for ZIKV HD78788. In urban-PP mosquitoes, we observed significantly higher viral loads for HD78788 (Figure 3A) on day 10 p.i. $(p<0.0001$ ) and day 14 p.i. $(p<0.0001)$ compared with both Asian ZIKVs, and on day 21 p.i. compared with FSS13025 ( $p=0.0007)$. In rural-KC mosquitoes, the viral loads of the saliva (Figure $3 \mathrm{~B}$ ) were significantly higher for HD78788 than for NC-2014-5132 on day 7 p.i. $(p=0.0021)$, day 10 p.i. $(p<0.0001)$, and day 14 p.i. $(p<0.0001)$, and higher than for FSS13025 on day 14 p.i. $(p<0.0001)$ and day 21 p.i. $(p=0.0034)$. Furthermore, the viral loads in the saliva of FSS13025-infected mosquitoes were significantly higher than for NC-2014-5132 on day 10 p.i. $(p=0.036)$ and day 14 p.i. $(p=0.0105)$. Finally, we observed a positive correlation between the transmission efficiency and viral load in the saliva (urban-PP: Figure 3C; rural-KC: Figure 3D; $\mathrm{r}^{2}>0.5$ and $p<0.0001$; Spearman correlation). 
A

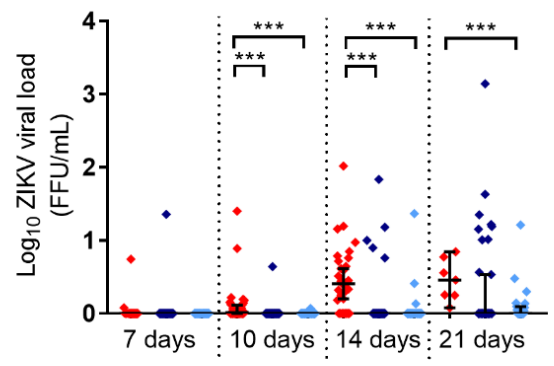

C

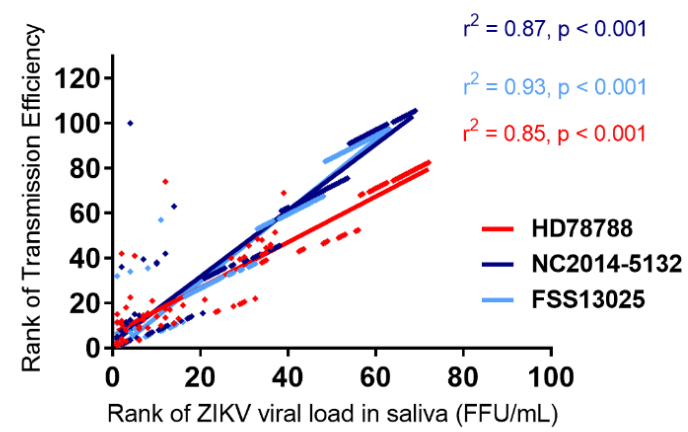

B

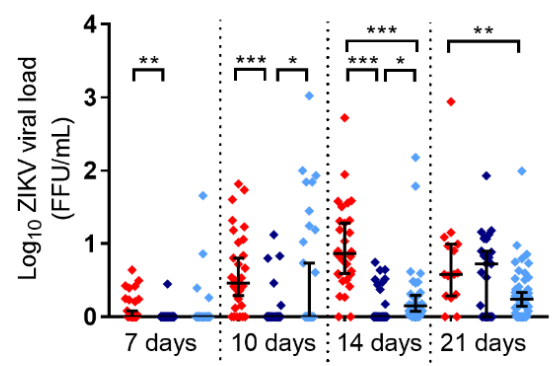

D

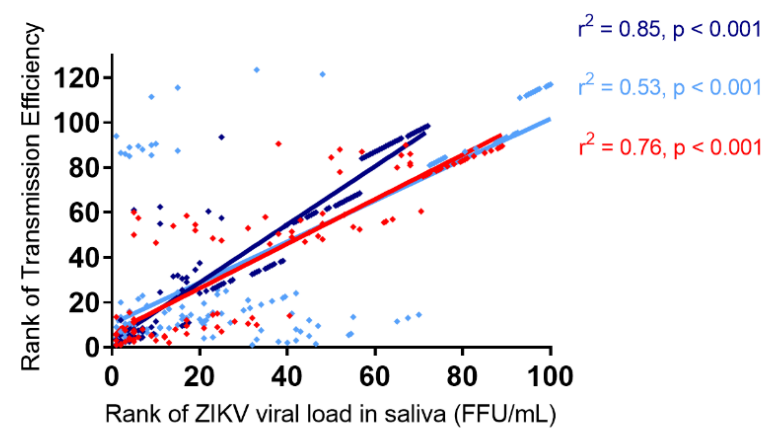

Figure 3. Viral load in the saliva of Ae. aegypti mosquitoes. Ae. aegypti populations from Phnom Penh (A,C) and Kampong Cham (B,D) were infected with ZIKV HD78788 (red), NC-2014-5132 (dark blue), or FSS13025 (light blue). The viral loads in saliva (A,B) were determined by RT-qPCR and the individual results are plotted with a median and 95\% CI. Statistical differences determined with Kruskal-Wallis test (Dunn's multiple comparison correction) are marked by asterisks: ${ }^{*} p<0.05$, ${ }^{* *} p<0.01$, and ${ }^{* *} p<0.001$. Correlation between the transmission efficiency and viral loads in saliva were evaluated by the Spearman correlation analysis (C,D).

\subsection{Survival Rate of ZIKV-Infected Mosquitoes}

During the vector competence study, we observed unusually high numbers of deaths for mosquitoes infected with HD78788, with a survival rate of $6.4 \%$ for urban-PP and $11.2 \%$ for rural-KC mosquitoes until day 21 p.i. (Supplementary Table S3). These survival rates were significantly lower than for urban-PP mosquitoes infected with NC-2014-5132 (18.1\%, $p=0.003)$ or FSS13025 (14.5\%, $p=0.0326)$, and rural-KC mosquitoes infected with FSS13025 $(28.4 \%, p=0.0005)$. Overall, the rate of survival was the highest in rural-KC mosquitoes infected with FSS13025 (28.4\% after 21 days p.i.). This was significantly higher compared with rural-KC mosquitoes infected with NC-2014-5132 $(13.1 \%, p=0.0038)$ and higher than the survival of urban-PP mosquitoes infected with FSS13025 (14.5\%, $p=0.0076)$. As these findings were unexpected, a comparison of the uninfected mosquitoes are not possible as we did not include a matched number of uninfected mosquitoes over the duration of the experiments.

\subsection{ZIKV Replication in Neonatal Swiss Mice}

The comparative analysis of the pathogenesis in mammals for the different ZIKV strains was done by infecting neonatal Swiss mice intracerebral and monitoring their weight (Figure 4A), general health condition (Supplementary Figure S3), and survival rate (Figure 4B) over 21 days or until death. All of the mice infected with HD78788 ( $n=20)$ died before the end of the experiment (between day 6 and 10 p.i.). Until day 6 p.i., these mice increased their weight similar to the mice infected with the Asian ZIKVs and the control. However, from day 6 p.i. onwards, the health of mice infected with African ZIKVs deteriorated until 10 days p.i. In contrast, the survival rates of mice infected with the Asian 
lineage strains were much higher, with mortality rates for NC-2014-5132 and FSS13025 infection of $6 \%$ and $15 \%$, respectively, at the end of experiment. In addition, the health of the mice was less affected as they only appeared weaker than the controls when infected with NC-2014-5132, or showed limb weakness when infected with FSS13025. The mice infected with FSS13025 exhibited more weight loss from day 14 p.i. onwards compared with the mice infected with NC-2014-5132 and the control mice.

A

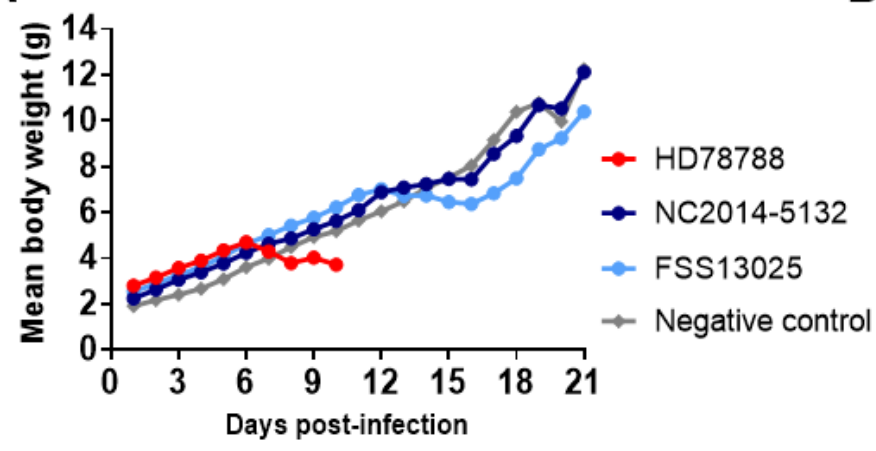

B

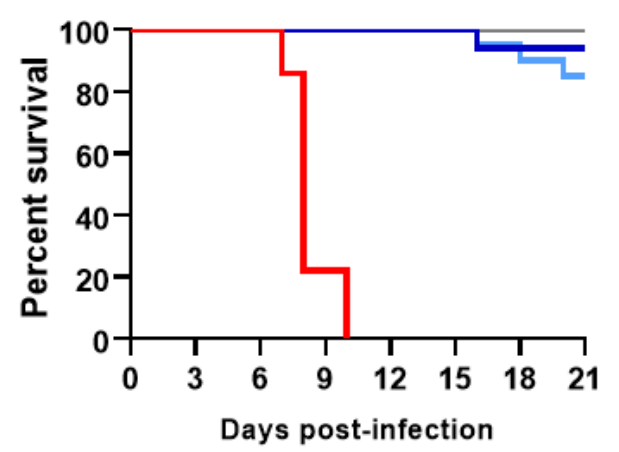

Figure 4. ZIKV infection in neonatal Swiss mice. Mice were infected with ZIKV HD78788 (red), NC-2014-5132 (dark blue), or FSS13025 (light blue) at the age of one day old. Body weight (A) was checked every day and survival was monitored for 21 days $(\mathbf{B})$.

The viral load in the blood and several organs was analyzed by RT-qPCR after death or euthanasia (Supplementary Figure S4, Supplementary Table S4). All of the ZIKVs were replicated in the blood and all of the tested organs, with the highest viral loads in the brain followed by the blood. The viral loads in blood and most organs were higher at day 7 p.i. and were significantly higher at day 8 and 10 p.i. for strain HD78788 than for NC-2014-5132 and FSS13025 (after euthanasia at day 7 and 8-10 p.i.). For HD78788, the viral load was the highest in the brain (median: 648, 3730, $1080 \mathrm{FFU} / \mathrm{mL}, 643-653,713-7720$, 361-16900 FFU/mL 95\% CI at day 7, 8-10 p.i, respectively) and the blood (median: 10, 1575, $642 \mathrm{FFU} / \mathrm{mL}, 6-14,3-7910,5-1280 \mathrm{FFU} / \mathrm{mL} 95 \% \mathrm{CI}$ at day 7, 8-10 p.i, respectively; Supplementary Table S4).

\section{Discussion}

We investigated the replication of different ZIKV lineages in vitro and in vivo and observed significant differences between the African lineage strain HD78788 and the Asian lineage strains NC-2014-5132 and FSS13025. The discrimination into the two lineages is based on the phylogenetic analysis and geographic origin [28]. The rapid spread and clinical presentation with severe neurological and congenital abnormalities raised the question of whether the Asian lineages are phenotypically different from the African viruses. Our growth analysis in simian and mosquito cells showed that the African virus (human isolate HD78788) was highly pathogenic and induced cell death in all cell lines. This was already reported [28-34] mainly with the African prototype virus MR766 isolated from the sentinel monkey in 1947 in Uganda [35]. However, this strain has a complex and heterogeneous passage history, and exists in at least three different variations [28] (Genbank NC012532, AY632535, HQ234498). Contrary to our in vitro results, the analysis in human endothelial cells found a faster replication and induction of CPE for the Asian lineage strains (PRVABC59 and FLR) when compared with African strains (MR766 and $\mathrm{IbH} 30656)$ [36]. Similar findings were reported from human cortical progenitor cells and in human brain organoids infected with a recent ZIKV isolate from Brazil (ZIKV ${ }^{\mathrm{BR}}$ ) compared with ZIKV MR766 [37].

In addition to the analysis of the growth in vitro, we compared the African and Asian viruses for their vector competence in two different populations of Cambodian Ae. aegypti mosquitoes (urban and rural). This is of special interest, as the reported circulation of 
ZIKV in Asia and especially in Cambodia is low $[4,16]$, which is partially explained by the low rate of symptomatic infections [38], the challenging diagnostics in a DENV endemic country [39], and the lack of a ZIKV-specific surveillance program. Therefore, vector competence of Cambodian mosquitoes for different ZIKV strains is highly valuable for risk assessment. Both Cambodian Ae. aegypti populations are competent ZIKV vectors with the virus present in saliva as early as 7 days p.i. Our results confirmed multiple former reports of Ae. aegypti in other countries and continents as highly competent to transmit ZIKV [10]. Our comparison of different Ae. aegypti populations revealed that transmission was observed earlier and in higher rates in the Kampong Cham mosquitoes originated from a rural habitat compared with the urban mosquito population from Phnom Penh. Significant differences in the ZIKV transmission were similarly found for Ae aegypti populations from Brazil, USA, and the Dominican Republic, where only the latter were able to transmit all of the investigated ZIKV strains [12]. In that same study, the African strain (DakAR41525) was able to infect, disseminate, and transmit in all of the investigated Ae. aegypti populations, whereas the Asian strains (FSS13025 and MEX1-7) showed significantly lower infection and dissemination rates in the Brazil and US mosquito populations. This is similar to our findings, where the African strain showed much higher dissemination rates. Furthermore, our results are in concordance with high ZIKV titers and transmission efficiency observed in Ae. aegypti saliva from New Caledonia infected with HD78788 compared with NC-2014-5132 [40]. However, we did not observe major differences in the infection and dissemination rates between the ZIKV lineages and Ae. aegypti populations, as we saw the most dissimilarities in the transmission. Differences in dissemination and transmission rates indicate that the different ZIKV strains might differ in their ability to cross the midgut and salivary gland infection and escape barriers. The ZIKV strains might also vary in their replication capacity in the mosquito midgut and other organs [41]. The differences between the ZIKV strains regarding their transmission can also be caused by the innate immunity of mosquitoes [42,43]. Additionally, intrinsic features of mosquito populations such as genetics may implicate in the interactions between mosquito and arboviruses, as was observed in a study in Thailand [44]. The authors observed that wild type Ae. aegypti vary in their infectious dose-response to oral DENV. Further investigation on the genetic susceptibility of the two mosquito populations is needed to decipher the observed difference in urban and rural mosquitoes in Cambodia.

Specific virus-mosquito interactions might play a role in the variation of survival rates we observed, depending on the mosquito population and the virus used for infection. We observed a higher pathogenicity in mosquitoes infected with the African strain HD78788. In addition, the urban mosquitoes had lower survival rates across all ZIKV strains compared with the rural mosquitoes. This indicates a co-evolution of the virus and vector in the rural environment, and that ZIKV might have circulated in Cambodia much earlier than 2007, as suggested by retrospective investigations [16], especially as phylogenetic analyses indicate the introduction of ZIKV to Southeast Asia as early as the $1950 \mathrm{~s}$ [8].

We further studied the pathogenesis of the different ZIKV lineages in the mouse model infecting neonatal Swiss mice via intracerebral injection. As seen before for the in vitro experiments, as well as for the mosquito vector competence, the African strain HD78788 showed a higher pathogenicity causing 100\% lethality within 10 days p.i. This finding is consistent with the results described by Fernandes et al. [45] who also performed this experiment on neonatal Swiss mice where they observed neurological symptoms within 6 days p.i. However, they used ZIKV strain SPH2015, which was isolated in Brazil in 2015. In another study, in 8-week-old interferon-deficient A129 mice infected with ZIKV strain H/PF/2013 (Asian lineage, isolated in 2013 in French Polynesia), a rapid onset of severe clinical signs and death within 7 days p.i. was observed [46]. These findings are similar to our results for the HD78788-infected mice. Moreover, in our study, the Asian lineage strains replicated to lower titers than the African strain and caused fewer clinical symptoms and lower mortality rates. We detected ZIKV viremia in the blood and brain with higher titers than in the other organs, which was also seen in the studies mentioned above. Again, 
the African strain HD78788 induced higher viral loads in mouse organs than the Asian strains, which reflected our results in cells and in mosquitoes. High replication, especially from African lineage ZIKV strains, was formerly reported from neuronal cell lines as well $[29,31,33,47]$. The generally less pathogenic phenotype of the Asian lineage viruses might lead to a less acute, but longer persisting infection. Persistence would allow the virus to infiltrate neuronal tissue and cause birth defects, whereas more lethal ZIKV strains (like the African lineage) might cause more miscarriages/stillbirths [48]. Although the Swiss mouse model is sufficient to investigate virus-depend pathogenicity and disease outcomes, other knock-out mice and immunocompetent humanized mouse models that allow ZIKV infection in adult mice through the natural peripheral route, e.g., the hSTAT2 [49] can be utilized to more accurately mimic natural Zika infection and for the development of therapeutics and vaccines.

We had no opportunity to include an American sub-lineage isolate in our comparative analysis. There might be substantial differences between the most recent outbreak viruses in the Americas and the isolates obtained from the epidemics on the Pacific islands or even older Asian isolates [50]. This was indicated in the vector competence of Ae. aegypti from Singapore, as the American isolate BEH815744 replicated faster and more successfully compared with the Asian ZIKV H/PF13 [51]. On the other hand, it is questionable whether the introduction of American ZIKV might lead to a wide distribution, as Asian ZIKVs are already circulating and a certain level of immunity exists in the population in Southeast Asia, even if its extent is hard to estimate as seroprevalence studies are missing.

\section{Conclusions}

Overall, our results confirm the different phenotypes of African and Asian ZIKV isolates in different in vitro and in vivo models. We showed that Cambodian Ae. aegypti mosquitoes are competent to transmit different ZIKV strains in particular rural mosquitoes, which is of interest for public health, as these mosquitoes are distributed widely across the country. However, the low transmission efficiency for Asian ZIKV strains, especially in the urban Ae. Aegypti, might be one factor contributing to the low circulation of ZIKV in Cambodia. Future studies should include American ZIKV strains to test if the introduction of this virus clade could lead to efficient transmission. Additionally, other possible vector species like Ae. albopictus should be tested for their vector competence, as this species is also widely distributed in Cambodia. Furthermore, the immunological status of the Cambodian population for ZIKV should be investigated as well as how the immunity for DENV might interfere with ZIKV infection. Our findings highlight the need for intensified, ZIKV-specific surveillance among the human population and vector species.

Supplementary Materials: The following are available online at https:/ / www.mdpi.com/article/ 10.3390/microorganisms9061250/s1. Figure S1: Phylogenetic tree of ZIKV strains based on partial nonstructural protein 5 gene sequences. Figure S2: Viral load of two different Cambodian Ae. aegypti mosquito populations. Figure S3: Health score in neonatal Swiss mice after ZIKV infection. Figure S4: Viral load ZIKV in different organs of neonatal Swiss mice. Table S1: Infection, dissemination, and transmission rates of Phnom Penh Aedes aegypti mosquito population. Table S2: Infection, dissemination, and transmission rates of Kampong Cham Aedes aegypti mosquito population. Table S3: Survival rates of ZIKV infected mosquitoes. Table S4: Viral load in organs of neonatal Swiss mice after death or euthanasia (21d p.i.).

Author Contributions: Conceptualization, M.D.-R. and V.D.; methodology, T.P.O., H.A., S.I., B.P., and V.D.; software, T.P.O. and H.A.; validation, V.D. and P.D.; formal analysis, T.P.O., H.A., B.P., S.B., and R.C.; investigation, T.P.O., H.A., S.I., B.P., S.P., S.B., and R.C.; resources, M.D.-R., P.D., and V.D.; data curation, T.P.O., B.P., and R.C.; writing-original draft preparation, T.P.O. and H.A.; writing-review and editing, P.D. and V.D.; supervision, P.D. and V.D.; project administration, P.D. and V.D.; funding acquisition, M.D.-R. and V.D. All authors have read and agreed to the published version of the manuscript. 
Funding: This work was supported by Institut Pasteur in Paris in the frame of the Actions Concertees Inter Pasteuriennes (ZikAe project, ACIP A-15-2014) and by the Institut Pasteur du Cambodge.

Institutional Review Board Statement: The study was conducted according to the World Animal Health Organization (OIE) guiding principles on animal welfare included in the OIE Terrestrial Animal Health Code. Mouse experiment were approved by the National Animal Health and Production Research Institute (NAHPRI) from General Directorate for Animal Health and Production (GDAHP), Ministry of Agriculture, Forestry, and Fisheries.

Informed Consent Statement: Written informed consent has been obtained from the blood donor volunteers before sampling.

Data Availability Statement: Publicly available datasets were analyzed in this study. This data can be found here: https:/ / drive.google.com/drive/folders/1-0OySA75-bw9nMq2uDhRH7m-9YrJ7v7q.

Acknowledgments: The authors thank Robert Tesh (World Reference Center for Emerging Viruses and Arboviruses at the University of Texas Medical Branch, Galveston, TX, USA) who kindly provided ZIKV FSS13025, Myrielle Dupont-Rouzeyrol (Institut Pasteur de Nouvelle-Calédonie) who kindly provided ZIKV NC-2014-5132, and Mawlouth Diallo and Oumar Faye (Institut Pasteur de Dakar) who kindly provided ZIKV HD78788. We thank Kimhuor Sour and Moeun Chhum from the Medical Entomology Unit for their technical assistance. The Aedes aegypti Aag2 cell line was kindly provided by Julien Pompon (Duke-NUS Graduate Medical School, Singapore). This work was supported by Institut Pasteur in Paris in the frame of the Actions Concertées Inter Pasteuriennes (ZikAe project, ACIP A-15-2014) and by the Institut Pasteur du Cambodge.

Conflicts of Interest: The authors declare no conflict of interest.

\section{References}

1. Dick, G.W.A.; Kitchen, S.F.; Haddow, A.J. Zika Virus (I). Isolations and Serological Specificity. Trans. R. Soc. Trop. Med. Hyg. 1952, 46, 509-520. [CrossRef]

2. Marchette, N.J.; Garcia, R.; Rudnick, A. Isolation of Zika Virus from Aedes Aegypti Mosquitoes in Malaysia. Am. J. Trop. Med. Hyg. 1969, 18, 411-415. [CrossRef] [PubMed]

3. Olson, J.G.; Ksiazek, T.G. Suhandiman; Triwibowo Zika Virus, a Cause of Fever in Central Java, Indonesia. Trans. R. Soc. Trop. Med. Hyg. 1981, 75, 389-393. [CrossRef]

4. Duong, V.; Dussart, P.; Buchy, P. Zika Virus in Asia. Int. J. Infect. Dis. 2017, 54, 121-128. [CrossRef] [PubMed]

5. Duffy, M.R.; Chen, T.-H.; Hancock, W.T.; Powers, A.M.; Kool, J.L.; Lanciotti, R.S.; Pretrick, M.; Marfel, M.; Holzbauer, S.; Dubray, C.; et al. Zika Virus Outbreak on Yap Island, Federated States of Micronesia. N. Engl. J. Med. 2009, 360, $2536-2543$. [CrossRef]

6. Heang, V.; Yasuda, C.Y.; Sovann, L.; Haddow, A.D.; Travassos da Rosa, A.P.; Tesh, R.B.; Kasper, M.R. Zika Virus Infection, Cambodia, 2010. Emerg. Infect. Dis. 2012, 18, 349-351. [CrossRef]

7. Sanchez, J.D. PAHO/WHO I Regional Zika Epidemiological Update (Americas). 25 August 2017. Available online: https: / / www.paho.org/hq/index.php?option=com_content\&view=article\&id=11599:regional-zika-epidemiological-updateamericas\&Itemid $=41691 \& l a n g=$ en (accessed on 11 October 2019).

8. Pettersson, J.H.-O.; Bohlin, J.; Dupont-Rouzeyrol, M.; Brynildsrud, O.B.; Alfsnes, K.; Cao-Lormeau, V.-M.; Gaunt, M.W.; Falconar, A.K.; de Lamballerie, X.; Eldholm, V.; et al. Re-Visiting the Evolution, Dispersal and Epidemiology of Zika Virus in Asia. Emerg. Microbes Infect. 2018, 7, 79. [CrossRef]

9. Wongsurawat, T.; Athipanyasilp, N.; Jenjaroenpun, P.; Jun, S.-R.; Kaewnapan, B.; Wassenaar, T.M.; Leelahakorn, N.; Angkasekwinai, N.; Kantakamalakul, W.; Ussery, D.W.; et al. Case of Microcephaly after Congenital Infection with Asian Lineage Zika Virus, Thailand. Emerg. Infect. Dis. J. 2018, 24, 1758-1761. [CrossRef]

10. Boyer, S.; Calvez, E.; Chouin-Carneiro, T.; Diallo, D.; Failloux, A.-B. An Overview of Mosquito Vectors of Zika Virus. Microbes Infect. 2018, 20, 646-660. [CrossRef]

11. Weger-Lucarelli, J.; Rückert, C.; Chotiwan, N.; Nguyen, C.; Luna, S.M.G.; Fauver, J.R.; Foy, B.D.; Perera, R.; Black, W.C.; Kading, R.C.; et al. Vector Competence of American Mosquitoes for Three Strains of Zika Virus. PLoS Negl. Trop. Dis. 2016, 10, e0005101. [CrossRef]

12. Roundy, C.M.; Azar, S.R.; Rossi, S.L.; Huang, J.H.; Leal, G.; Yun, R.; Fernandez-Salas, I.; Vitek, C.J.; Paploski, I.A.D.; Kitron, U.; et al. Variation in Aedes Aegypti Mosquito Competence for Zika Virus Transmission. Emerg. Infect. Dis. 2017, $23,625-632$. [CrossRef]

13. Ciota, A.T.; Bialosuknia, S.M.; Zink, S.D.; Brecher, M.; Ehrbar, D.J.; Morrissette, M.N.; Kramer, L.D. Effects of Zika Virus Strain and Aedes Mosquito Species on Vector Competence. Emerg. Infect. Dis. 2017, 23, 1110-1117. [CrossRef] 
14. Pena, L.J.; Miranda Guarines, K.; Duarte Silva, A.J.; Sales Leal, L.R.; Mendes Félix, D.; Silva, A.; de Oliveira, S.A.; Junqueira Ayres, C.F.; Júnior, A.S.; de Freitas, A.C. In Vitro and in Vivo Models for Studying Zika Virus Biology. J. Gen. Virol. 2018, 99, 1529-1550. [CrossRef] [PubMed]

15. Bradley, M.P.; Nagamine, C.M. Animal Models of Zika Virus. Comp. Med. 2017, 67, 242-252. [PubMed]

16. Duong, V.; Ong, S.; Leang, R.; Huy, R.; Ly, S.; Mounier, U.; Ou, T.; In, S.; Peng, B.; Ken, S.; et al. Low Circulation of Zika Virus, Cambodia, 2007-2016. Emerg. Infect. Dis. 2017, 23, 296-299. [CrossRef]

17. Huy, R.; Buchy, P.; Conan, A.; Ngan, C.; Ong, S.; Ali, R.; Duong, V.; Yit, S.; Ung, S.; Te, V.; et al. National Dengue Surveillance in Cambodia 1980-2008: Epidemiological and Virological Trends and the Impact of Vector Control. Bull. World Health Organ. 2010, 88, 650-657. [CrossRef]

18. Faye, O.; Freire, C.C.M.; Iamarino, A.; Faye, O.; de Oliveira, J.V.C.; Diallo, M.; Zanotto, P.M.A.; Sall, A.A. Molecular Evolution of Zika Virus during Its Emergence in the 20th Century. PLoS Negl. Trop. Dis. 2014, 8, e2636. [CrossRef] [PubMed]

19. Stojanovich, C.J.; Scott, H.G.; Stojanovich, C.J.; Communicable Disease Center (U.S.) ; United States Army; Special Epidemiologic Team. Illustrated Key to Aedes Mosquitoes of Vietnam; U.S. Department of Health, Education, and Welfare, Public Health Service, Communicable Disease Center: Atlanta, GA, USA, 1965.

20. Reuben, R.; Hiriyan, J.; Akiyama, J. Illustrated Keys to Species of Culex (Culex) Associated with Japanese Encephalitis in Southeast Asia (Diptera: Culicidae). Mosq. Syst. 1994, 26, 75-96.

21. Rattanarithikul, R.; Harbach, R.; Harrison, B.; Panthusiri, P.; Jones, J.; Coleman, R. Illustrated Keys to the Mosquitoes of Thailand II. Genera Culex and Lutzia. Southeast Asian J. Trop. Med. Public Health 2005, 36, 1-97.

22. Kumar, M.; Kumar, P. Euthanasia Procedure Used in Experimental Laboratory. In Animal Models of Neurological Disorders: Principle and Working Procedure for Animal Models of Neurological Disorders; Springer: Singapore, 2017; pp. 307-314. ISBN 978-981-10-5980-3.

23. Ou, T.P.; Yun, C.; Auerswald, H.; In, S.; Leang, R.; Huy, R.; Choeung, R.; Dussart, P.; Duong, V. Improved Detection of Dengue and Zika Viruses Using Multiplex RT-QPCR Assays. J. Virol. Methods 2020, 282, 113862. [CrossRef] [PubMed]

24. OIE-World Organisation for Animal Health. Available online: https: / www.oie.int/index.php?id=169\&L=0\&htmfile=chapitre _ aw_research_education.htm (accessed on 27 January 2020).

25. Kay, B.H.; Prakash, G.; Andre, R.G. Aedes Albopictus and Other Aedes (Stegomyia) Species in Fiji. J. Am. Mosq. Control. Assoc. 1995, 11, 230-234.

26. Vicenti, I.; Boccuto, A.; Giannini, A.; Dragoni, F.; Saladini, F.; Zazzi, M. Comparative Analysis of Different Cell Systems for Zika Virus (ZIKV) Propagation and Evaluation of Anti-ZIKV Compounds in Vitro. Virus Res. 2018, 244, 64-70. [CrossRef] [PubMed]

27. Saito, K.; Fukasawa, M.; Shirasago, Y.; Suzuki, R.; Osada, N.; Yamaji, T.; Wakita, T.; Konishi, E.; Hanada, K. Comparative Characterization of Flavivirus Production in Two Cell Lines: Human Hepatoma-Derived Huh7.5.1-8 and African Green Monkey Kidney-Derived Vero. PLoS ONE 2020, 15, e0232274. [CrossRef]

28. Haddow, A.D.; Schuh, A.J.; Yasuda, C.Y.; Kasper, M.R.; Heang, V.; Huy, R.; Guzman, H.; Tesh, R.B.; Weaver, S.C. Genetic Characterization of Zika Virus Strains: Geographic Expansion of the Asian Lineage. PLoS Negl. Trop. Dis. 2012, 6, e1477. [CrossRef] [PubMed]

29. Anfasa, F.; Siegers, J.Y.; van der Kroeg, M.; Mumtaz, N.; Stalin Raj, V.; de Vrij, F.M.S.; Widagdo, W.; Gabriel, G.; Salinas, S.; Simonin, Y.; et al. Phenotypic Differences between Asian and African Lineage Zika Viruses in Human Neural Progenitor Cells. mSphere 2017, 2, e00292-17. [CrossRef] [PubMed]

30. Bowen, J.R.; Quicke, K.M.; Maddur, M.S.; O'Neal, J.T.; McDonald, C.E.; Fedorova, N.B.; Puri, V.; Shabman, R.S.; Pulendran, B.; Suthar, M.S. Zika Virus Antagonizes Type I Interferon Responses during Infection of Human Dendritic Cells. PLoS Pathog. 2017, 13, e1006164. [CrossRef] [PubMed]

31. Gabriel, E.; Ramani, A.; Karow, U.; Gottardo, M.; Natarajan, K.; Gooi, L.M.; Goranci-Buzhala, G.; Krut, O.; Peters, F.; Nikolic, M.; et al. Recent Zika Virus Isolates Induce Premature Differentiation of Neural Progenitors in Human Brain Organoids. Cell Stem Cell 2017, 20, 397-406.e5. [CrossRef]

32. Sheridan, M.A.; Yunusov, D.; Balaraman, V.; Alexenko, A.P.; Yabe, S.; Verjovski-Almeida, S.; Schust, D.J.; Franz, A.W.; Sadovsky, Y.; Ezashi, T.; et al. Vulnerability of Primitive Human Placental Trophoblast to Zika Virus. Proc. Natl. Acad. Sci. USA 2017, 114, e1587-e1596. [CrossRef] [PubMed]

33. Zhang, F.; Hammack, C.; Ogden, S.C.; Cheng, Y.; Lee, E.M.; Wen, Z.; Qian, X.; Nguyen, H.N.; Li, Y.; Yao, B.; et al. Molecular Signatures Associated with ZIKV Exposure in Human Cortical Neural Progenitors. Nucleic Acids Res. 2016, 44, 8610-8620. [CrossRef]

34. McGrath, E.L.; Rossi, S.L.; Gao, J.; Widen, S.G.; Grant, A.C.; Dunn, T.J.; Azar, S.R.; Roundy, C.M.; Xiong, Y.; Prusak, D.J.; et al. Differential Responses of Human Fetal Brain Neural Stem Cells to Zika Virus Infection. Stem Cell Rep. 2017, 8, 715-727. [CrossRef]

35. Weaver, S.C.; Costa, F.; Garcia-Blanco, M.A.; Ko, A.I.; Ribeiro, G.S.; Saade, G.; Shi, P.-Y.; Vasilakis, N. Zika Virus: History, Emergence, Biology, and Prospects for Control. Antivir. Res. 2016, 130, 69-80. [CrossRef] [PubMed]

36. Liu, S.; DeLalio, L.J.; Isakson, B.E.; Wang, T.T. AXL-Mediated Productive Infection of Human Endothelial Cells by Zika Virus. Circ. Res. 2016, 119, 1183-1189. [CrossRef]

37. Cugola, F.R.; Fernandes, I.R.; Russo, F.B.; Freitas, B.C.; Dias, J.L.M.; Guimarães, K.P.; Benazzato, C.; Almeida, N.; Pignatari, G.C.; Romero, S.; et al. The Brazilian Zika Virus Strain Causes Birth Defects in Experimental Models. Nature 2016, 534, $267-271$. [CrossRef] [PubMed] 
38. Moghadas, S.M.; Shoukat, A.; Espindola, A.L.; Pereira, R.S.; Abdirizak, F.; Laskowski, M.; Viboud, C.; Chowell, G. Asymptomatic Transmission and the Dynamics of Zika Infection. Sci. Rep. 2017, 7, 51-829. [CrossRef] [PubMed]

39. Heinz, F.X.; Stiasny, K. The Antigenic Structure of Zika Virus and Its Relation to Other Flaviviruses: Implications for Infection and Immunoprophylaxis. Microbiol. Mol. Biol. Rev. 2017, 81, e00055-16. [CrossRef] [PubMed]

40. Calvez, E.; O'Connor, O.; Pol, M.; Rousset, D.; Faye, O.; Richard, V.; Tarantola, A.; Dupont-Rouzeyrol, M. Differential Transmission of Asian and African Zika Virus Lineages by Aedes Aegypti from New Caledonia. Emerg. Microbes Infect. 2018, 7, 159. [CrossRef]

41. Franz, A.W.E.; Kantor, A.M.; Passarelli, A.L.; Clem, R.J. Tissue Barriers to Arbovirus Infection in Mosquitoes. Viruses 2015, 7, 3741-3767. [CrossRef]

42. Blair, C.D. Mosquito RNAi Is the Major Innate Immune Pathway Controlling Arbovirus Infection and Transmission. Future Microbiol. 2011, 6, 265-277. [CrossRef]

43. Sim, S.; Jupatanakul, N.; Dimopoulos, G. Mosquito Immunity against Arboviruses. Viruses 2014, 6, 4479-4504. [CrossRef]

44. Pongsiri, A.; Ponlawat, A.; Thaisomboonsuk, B.; Jarman, R.G.; Scott, T.W.; Lambrechts, L. Differential Susceptibility of Two Field Aedes Aegypti Populations to a Low Infectious Dose of Dengue Virus. PLoS ONE 2014, 9, e92971. [CrossRef]

45. Fernandes, N.C.C.A.; Nogueira, J.S.; Réssio, R.A.; Cirqueira, C.S.; Kimura, L.M.; Fernandes, K.R.; Cunha, M.S.; Souza, R.P.; Guerra, J.M. Experimental Zika Virus Infection Induces Spinal Cord Injury and Encephalitis in Newborn Swiss Mice. Exp. Toxicol. Pathol. 2017, 69, 63-71. [CrossRef] [PubMed]

46. Aliota, M.T.; Caine, E.A.; Walker, E.C.; Larkin, K.E.; Camacho, E.; Osorio, J.E. Characterization of Lethal Zika Virus Infection in AG129 Mice. PLoS Negl. Trop. Dis. 2016, 10, e0004682. [CrossRef] [PubMed]

47. Simonin, Y.; Loustalot, F.; Desmetz, C.; Foulongne, V.; Constant, O.; Fournier-Wirth, C.; Leon, F.; Molès, J.-P.; Goubaud, A.; Lemaitre, J.-M.; et al. Zika Virus Strains Potentially Display Different Infectious Profiles in Human Neural Cells. EBioMedicine 2016, 12, 161-169. [CrossRef] [PubMed]

48. Udenze, D.; Trus, I.; Berube, N.; Gerdts, V.; Karniychuk, U. The African Strain of Zika Virus Causes More Severe in Utero Infection than Asian Strain in a Porcine Fetal Transmission Model. Emerg. Microbes Infect. 2019, 8, 1098-1107. [CrossRef]

49. Gorman, M.J.; Caine, E.A.; Zaitsev, K.; Begley, M.C.; Weger-Lucarelli, J.; Uccellini, M.B.; Tripathi, S.; Morrison, J.; Yount, B.L.; Dinnon, K.H.; et al. An Immunocompetent Mouse Model of Zika Virus Infection. Cell Host. Microbe 2018, 23, 672-685. [CrossRef]

50. Hu, T.; Li, J.; Carr, M.J.; Duchêne, S.; Shi, W. The Asian Lineage of Zika Virus: Transmission and Evolution in Asia and the Americas. Virol Sin 2019, 34, 1-8. [CrossRef]

51. Pompon, J.; Morales-Vargas, R.; Manuel, M.; Huat Tan, C.; Vial, T.; Hao Tan, J.; Sessions, O.M.; da Vasconcelos, P.C.; Ng, L.C.; Missé, D.A. Zika Virus from America Is More Efficiently Transmitted than an Asian Virus by Aedes Aegypti Mosquitoes from Asia. Sci. Rep. 2017, 7, 1-8. [CrossRef] 